Supporting Information

\title{
A Universal Method to Fabricate Transition Metal Single-atom Anchored Carbon with Excellent Oxygen Reduction Reaction Activity
}

Lin-Qian Yu, Wen-Jing Xia, Wen-Jie Ma, Te-Er Wen, Shu-La Chen, Feng Jin, Bao-Cheng Huang*, and Ren-Cun Jin

Laboratory of Water Pollution Remediation, School of Life and Environmental Sciences, Hangzhou Normal University, Hangzhou 311121, China

*Corresponding author: Dr. Bao-Cheng Huang, E-mail: huangbc@hznu.edu.cn

This supporting information contains 11-page document, including 6 tables, 4 figures, and this cover page. 
Table $\mathrm{S} 1 \mathrm{Ca}$ and $\mathrm{Cu}$ contents of $0.1 \mathrm{Cu}-\mathrm{SAC} / \mathrm{N}$ and $\mathrm{AG}-\mathrm{Cu}-0.1$ by ICP tests (wt.\%).

\begin{tabular}{lll}
\hline & $\mathrm{Ca}$ & $\mathrm{Cu}$ \\
\hline $0.1 \mathrm{Cu}-\mathrm{SAC} / \mathrm{N}$ & $0.01 \%$ & $0.23 \%$ \\
$\mathrm{AG}-\mathrm{Cu}-0.1$ & $0.40 \%$ & $14.58 \%$ \\
\hline
\end{tabular}


Table S2 Organic element analysis of $\mathrm{AG} / \mathrm{N}, 0.1 \mathrm{Cu}-\mathrm{SAC} / \mathrm{N}, 0.05 \mathrm{Cu}-\mathrm{SAC} / \mathrm{N}$, $0.1 \mathrm{Cu}-\mathrm{SAC} / \mathrm{N}$, and $0.2 \mathrm{Cu}-\mathrm{SAC} / \mathrm{N}$.

\begin{tabular}{lllll}
\hline & N & C & H & O \\
\hline AG/N & $2.81 \pm 0.03 \%$ & $77.95 \pm 0.02 \%$ & $1.22 \pm 0.10 \%$ & $18.35 \pm 3.89 \%$ \\
$0.1 \mathrm{Cu}-\mathrm{SAC}$ & $0.12 \pm 0.02 \%$ & $79.57 \pm 0.07 \%$ & $0.92 \pm 0.22 \%$ & $14.63 \pm 0.34 \%$ \\
$0.2 \mathrm{Cu}-\mathrm{SAC} / \mathrm{N}$ & $4.67 \pm 0.05 \%$ & $79.53 \pm 0.07 \%$ & $1.55 \pm 0.06 \%$ & $13.95 \pm 0.12 \%$ \\
$0.1 \mathrm{Cu}-\mathrm{SAC} / \mathrm{N}$ & $5.25 \pm 0.06 \%$ & $77.01 \pm 0.07 \%$ & $1.48 \pm 0.01 \%$ & $15.10 \pm 0.27 \%$ \\
$0.05 \mathrm{Cu}-\mathrm{SAC} / \mathrm{N}$ & $3.73 \pm 0.05 \%$ & $81.86 \pm 0.07 \%$ & $1.48 \pm 0.00 \%$ & $12.45 \pm 0.17 \%$ \\
\hline
\end{tabular}


Table S3 BET specific surface area of $\mathrm{AG} / \mathrm{N}, 0.1 \mathrm{Cu}-\mathrm{SAC} / \mathrm{N}, 0.05 \mathrm{Cu}-\mathrm{SAC} / \mathrm{N}$, $0.1 \mathrm{Cu}-\mathrm{SAC} / \mathrm{N}$, and $0.2 \mathrm{Cu}-\mathrm{SAC} / \mathrm{N}$.

\begin{tabular}{ll}
\hline & BET Specific surface Area $\left(\mathbf{m}^{2} \cdot \mathbf{g}^{-1}\right)$ \\
\hline AG/N & 404.2 \\
$0.1 \mathrm{Cu}-\mathrm{SAC}$ & 873.0 \\
$0.2 \mathrm{Cu}-\mathrm{SAC} / \mathrm{N}$ & 1565.1 \\
$0.1 \mathrm{Cu}-\mathrm{SAC} / \mathrm{N}$ & 1551.9 \\
$0.05 \mathrm{Cu}-\mathrm{SAC} / \mathrm{N}$ & 954.4 \\
\hline
\end{tabular}


Table S4 The percentage of $\mathrm{C}, \mathrm{N}, \mathrm{O}$ in $0.05 \mathrm{Cu}-\mathrm{SAC} / \mathrm{N}, 0.1 \mathrm{Cu}-\mathrm{SAC} / \mathrm{N}$, and $0.2 \mathrm{Cu}-\mathrm{SAC} / \mathrm{N}$ by XPS fitting.

\begin{tabular}{lllll}
\hline & & $\mathbf{0 . 1 C u}-\mathbf{S A C} / \mathbf{N}$ & $\mathbf{0 . 0 5 C u - S A C} / \mathbf{N}$ & $\mathbf{0 . 2 C u - S A C / N}$ \\
\hline & C-C/C=C & $65.71 \%$ & $67.90 \%$ & $63.71 \%$ \\
$\mathrm{C}$ & C-O/C-N & $19.20 \%$ & $17.69 \%$ & $20.81 \%$ \\
& COOR & $15.09 \%$ & $14.40 \%$ & $15.49 \%$ \\
& Pyridinic N & $44.26 \%$ & $37.86 \%$ & $33.84 \%$ \\
N & Pyrrolic N & $46.44 \%$ & $34.10 \%$ & $28.63 \%$ \\
& Graphitic N & $11.30 \%$ & $28.05 \%$ & $37.53 \%$ \\
& C $=\mathrm{O}$ & $24.00 \%$ & $94.38 \%$ & $8.85 \%$ \\
\hline
\end{tabular}


Table S5 Onset potential (at $-0.5 \mathrm{~mA} \cdot \mathrm{cm}^{-2}$ ), half wave potential, and limited current density of $\mathrm{Pt} / \mathrm{C}, \mathrm{AG} / \mathrm{N}, 0.1 \mathrm{Cu}-\mathrm{SAC}, 0.05 \mathrm{Cu}-\mathrm{SAC} / \mathrm{N}, 0.1 \mathrm{Cu}-\mathrm{SAC} / \mathrm{N}$, and $0.2 \mathrm{Cu}-\mathrm{SAC} / \mathrm{N}$.

\begin{tabular}{llll}
\hline & Onset potential/V & $\begin{array}{l}\text { Half } \\
\text { potential/V }\end{array}$ & $\begin{array}{l}\text { Limiting } \\
\text { density }\left(\mathbf{m A} \mathbf{A} \cdot \mathbf{c m}^{-2}\right)\end{array}$ \\
\hline $\mathrm{Pt} / \mathrm{C}$ & 0.93 & 0.83 & 5.10 \\
$\mathrm{AG} / \mathrm{N}$ & 0.84 & 0.71 & 3.60 \\
$0.1 \mathrm{Cu}-\mathrm{SAC}$ & 0.77 & 0.66 & 3.02 \\
$0.2 \mathrm{Cu}-\mathrm{SAC} / \mathrm{N}$ & 0.91 & 0.78 & 4.54 \\
$0.1 \mathrm{Cu}-\mathrm{SAC} / \mathrm{N}$ & 0.90 & 0.80 & 5.05 \\
$0.05 \mathrm{Cu}-\mathrm{SAC} / \mathrm{N}$ & 0.85 & 0.69 & 3.60 \\
\hline
\end{tabular}


Table S6 Onset potential (at $-0.5 \mathrm{~mA} \cdot \mathrm{cm}^{-2}$ ), half wave potential, and limited current density of $\mathrm{Pt} / \mathrm{C}, 0.1 \mathrm{Cu}-\mathrm{SAC} / \mathrm{N}$ before and after 3000 cycles of $\mathrm{CV}$ test.

\begin{tabular}{lllllll}
\hline & $\begin{array}{l}\text { Limited current } \\
\text { density }\left(\mathbf{m A} \cdot \mathbf{c m}^{-2}\right)\end{array}$ & \multicolumn{2}{l}{ Half } & \multicolumn{2}{c}{ wave } & \multicolumn{2}{l}{ Onset potential/V } \\
& Initial & After & Initial & After & Initial & After \\
& 5.1 & 4.69 & 0.83 & 0.8 & 0.93 & 0.88 \\
$\mathrm{Pt} / \mathrm{C}$ & 5.05 & 4.58 & 0.8 & 0.79 & 0.90 & 0.90 \\
\hline
\end{tabular}




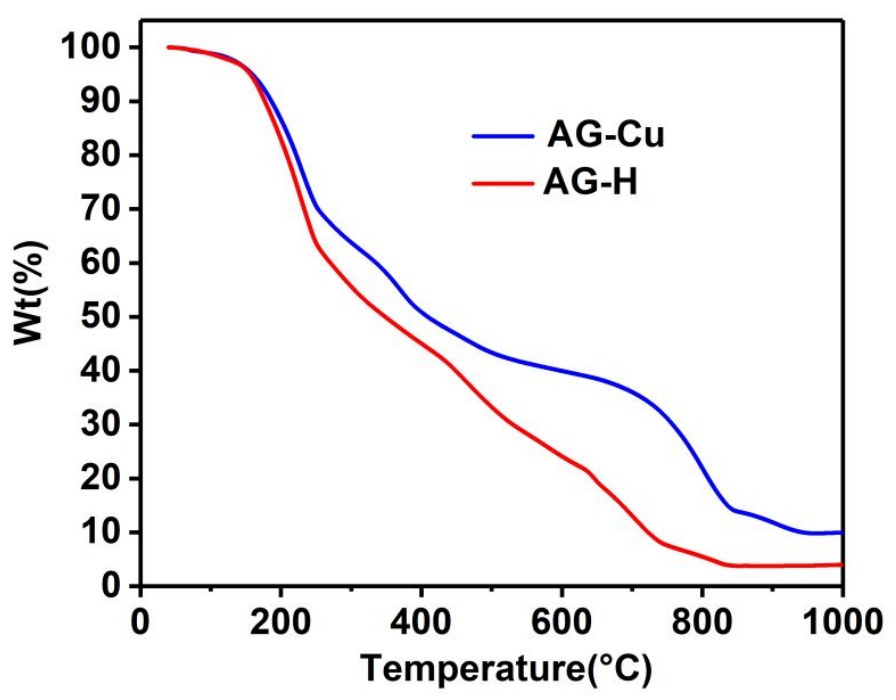

Figure S1. TGA analysis of $\mathrm{Cu}$ alginate (AG-Cu) and alginate hydrogel (AG-H). 

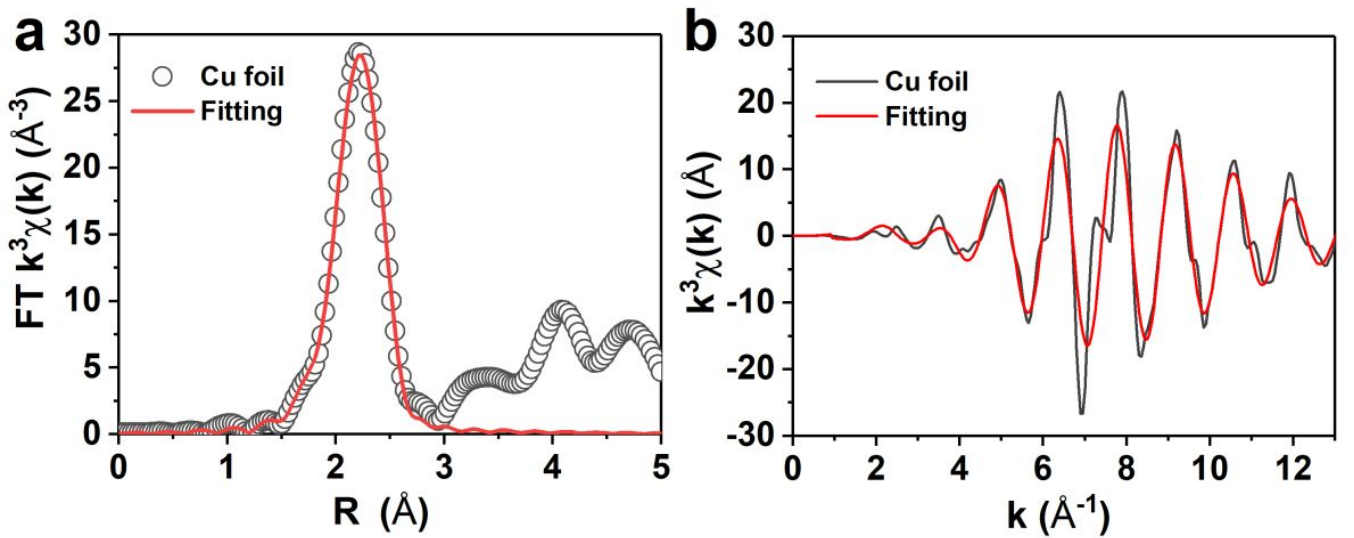

Figure S2. Fourier transform of K-edge (a) and R space (b) EXAFS spectra and fitting curves of $\mathrm{Cu}$ foil. 


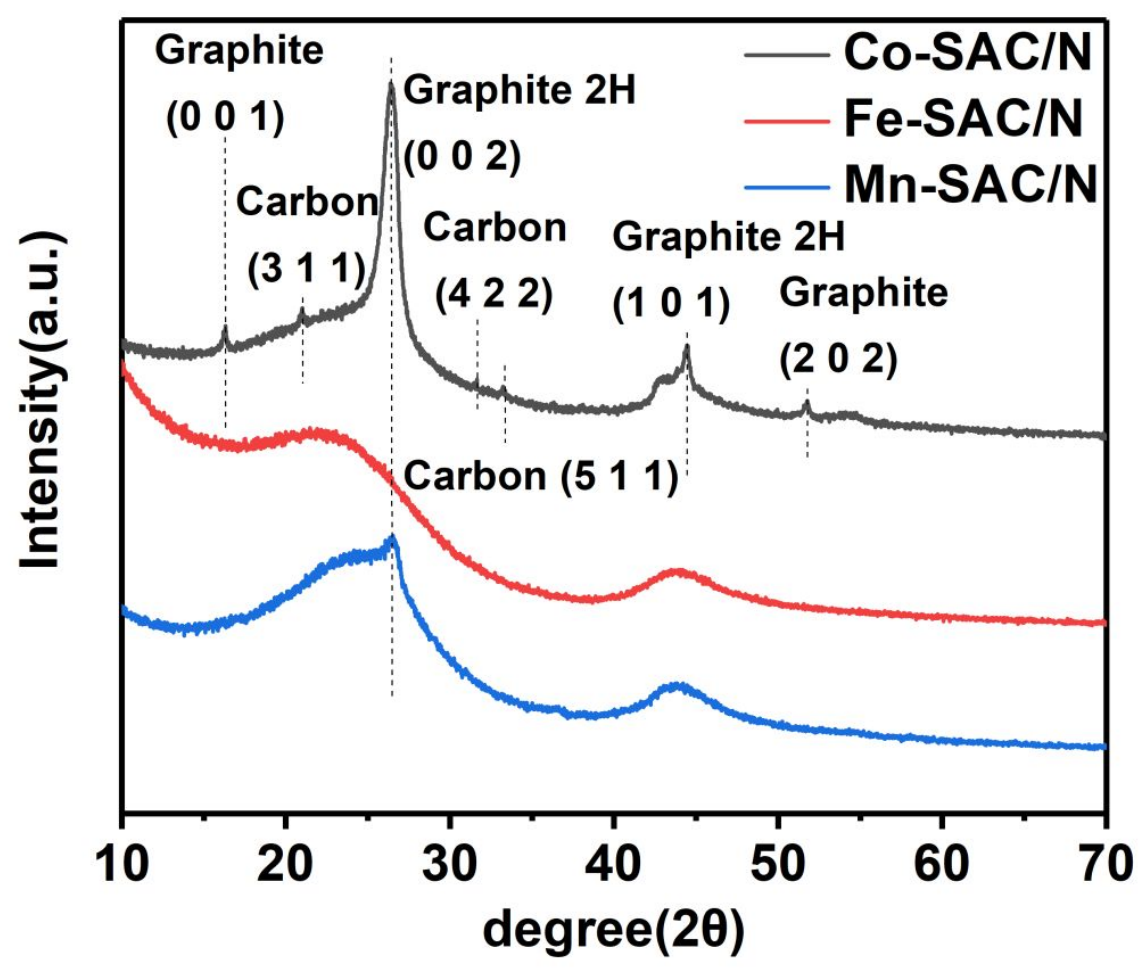

Figure S3. XRD patterns of Co-SAC/N, Fe-SAC/N, and Mn-SAC/N. 


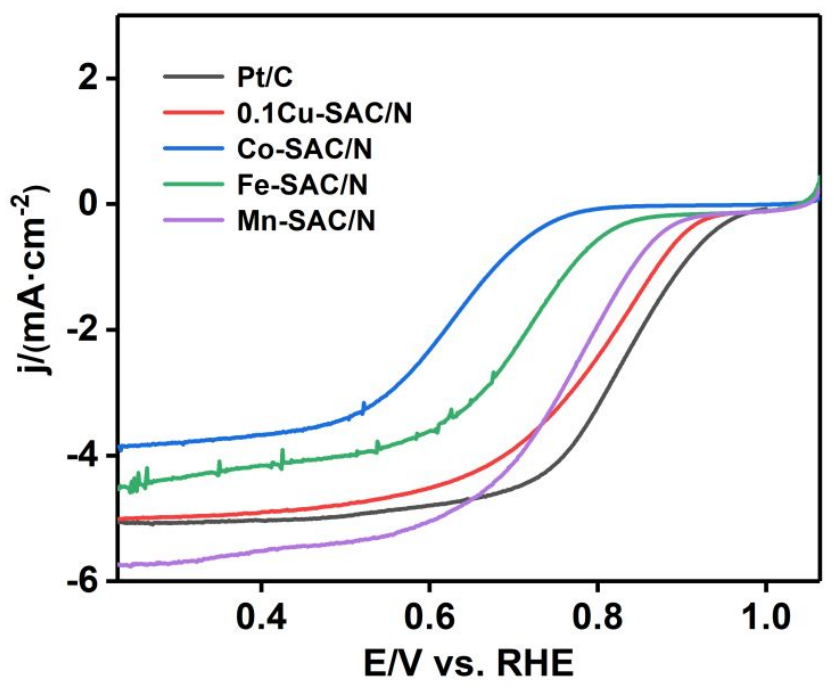

Figure S4. LSV curves of $\mathrm{Pt} / \mathrm{C}$ and as-prepared catalysts in $0.1 \mathrm{M} \mathrm{KOH}$. 
